\title{
Toward instructional design principles: Inducing Faraday's law with contrasting cases
}

\author{
Eric Kuo and Carl E. Wieman \\ Department of Physics and Graduate School of Education, \\ Stanford University, Stanford, California 94305, USA \\ (Received 11 October 2015; published 5 April 2016)
}

\begin{abstract}
Although physics education research (PER) has improved instructional practices, there are not agreed upon principles for designing effective instructional materials. Here, we illustrate how close comparison of instructional materials could support the development of such principles. Specifically, in discussion sections of a large, introductory physics course, a pair of studies compare two instructional strategies for teaching a physics concept: having students (i) explain a set of contrasting cases or (ii) apply and build on previously learned concepts. We compare these strategies for the teaching of Faraday's law, showing that explaining a set of related contrasting cases not only improves student performance on Faraday's law questions over building on a previously learned concept (i.e., Lorentz force), but also prepares students to better learn subsequent topics, such as Lenz's law. These differences persist to the final exam. We argue that early exposure to contrasting cases better focuses student attention on a key feature related to both concepts: change in magnetic flux. Importantly, the benefits of contrasting cases for both learning and enjoyment are enhanced for students who did not first attend a Faraday's law lecture, consistent with previous research suggesting that being told a solution can circumvent the benefits of its discovery. These studies illustrate an experimental approach for understanding how the structure of activities affects learning and performance outcomes, a first step toward design principles for effective instructional materials.
\end{abstract}

DOI: 10.1103/PhysRevPhysEducRes.12.010128

\section{TOWARD PRINCIPLES FOR EFFECTIVE INSTRUCTIONAL DESIGN}

Physics education research (PER) has built a research base for improved instruction of physics concepts in several ways. One branch of this effort focuses on assessment, producing conceptual tests that can be widely used, e.g., Refs. [1-3]. Another area focuses on students' prior knowledge by researching common student difficulties and developing activities designed to address them, e.g., Refs. [4,5]. A third area evaluates the efficacy of new instructional materials and approaches, often in comparison to traditional "lecture-and-practice" style instruction, e.g., Ref. [6]. These approaches have clarified valuable goals of physics instruction and have produced instruction for better achieving those goals.

One area not covered by these efforts is the development of knowledge for how to design effective instructional materials. While there is general agreement that instruction should support "active learning" and "interactive engagement," the different materials designed for interactive engagement can vary. Moreover, differences between various instructional settings, pedagogical approaches, materials, and assessments make identification of important features of instructional materials from existing studies difficult.

Published by the American Physical Society under the terms of the Creative Commons Attribution 3.0 License. Further distribution of this work must maintain attribution to the author $(s)$ and the published article's title, journal citation, and DOI.
In this paper, we work toward filling this gap, continuing a thread of work investigating how fine-grained differences in the structure of instructional materials can lead to different learning and performance outcomes [7]. Specifically, we compare two approaches to teaching a physics concept: (i) having students explain a set of examples illustrating the concept or (ii) having students connect the concept to a previously learned one. The first approach represents an effective strategy developed from cognitive psychology-using contrasting cases to help students notice important features. The second approach represents a common instructional practice-leveraging the sequence of topics to build new concepts from old ones, highlighting the coherence between different ideas in physics. For an instructor or curriculum designer creating materials for an active-engagement classroom, both approaches represent reasonable instructional possibilities. By comparing these two approaches to teaching the same physics concept (Faraday's law) in the same instructional setting (discussion sections of a large lecture course), we aim towards developing fine-grained, empiricallygrounded principles for effective instructional design.

\section{THE LEARNING BENEFITS OF CONTRASTING CASES}

\section{A. Highlighting key features}

Figure 1 contains three examples of wire loops in magnetic fields, where a current either is or is not induced. Imagine a physics student trying to explain when there will 


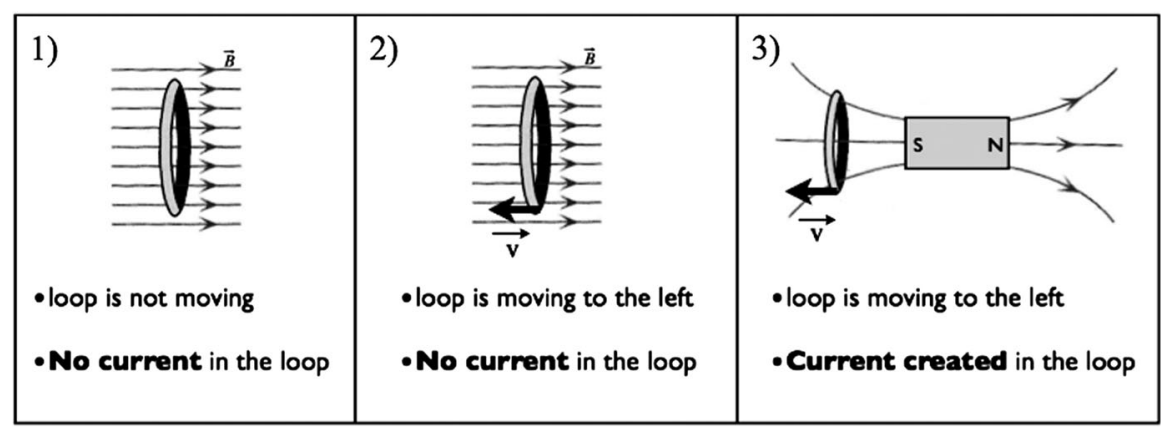

FIG. 1. A set of contrasting cases of wire loops in magnetic fields (adapted from the University of Washington Tutorials in Introductory Physics [8]).

be an induced current. From case 1 alone, many different simple rules are plausible. A student could propose that there is no current in the loop when there is no motion. Another possible explanation for case 1 is that there is no induced current when the loop is in a uniform magnetic field. While these explanations are part of the correct explanation, they are incomplete and not generally applicable. These answers would not be evidence that students are attending to the change in magnetic flux through the loop as the key explanatory factor.

Imagine the same student, but now given cases 1 and 2 . Comparing across the two, they could conclude that movement alone is not a sufficient condition to induce a current. However, it is still not clear what features can cause an induced current. By comparing the third case to the previous two, the importance of a nonuniform magnetic field becomes salient. Here, though a student's explanation of induced currents with these three examples alone may be far from complete, being forced to explain multiple examples can push the student's explanation to align more with Faraday's law. The contrasts between the cases help to differentiate the important features of the phenomenon [9], and prior research has illustrated the benefits of contrasting cases for helping students identify the deep structural features of unfamiliar phenomena [10-12].

Even after instruction, students commonly determine whether or not a current is induced by attending to the motion of wire loops and magnets, without considering whether or not the motion changes the magnetic flux [2]. Consistent with previous work in psychology, one would expect that the task of coming up with a general explanation may push students to look beyond these attractive, idiosyncratic surface features $[11,13]$.

\section{B. Preparation for future learning}

In explaining the induced current examples, it is possible to explain all the cases sufficiently and never spontaneously generate a magnetic flux-based explanation of induced currents. Are the contrasting cases instructionally useful if students do not invent the correct solution?
There is evidence that inventing with contrasting cases has benefits beyond directly discovering the principle at hand. Attempts to invent explanations of a set of contrasting cases can prepare students for future learning from subsequent direct instruction [14-16]. Schwartz and Martin provide one example [15]: students tasked with inventing a method for comparing data across different statistical distributions later learned a procedure for calculating standardized scores better than students who were initially told how to compare the sample data. Importantly, the students who invented failed to produce the correct procedure. Yet, by first seeking a solution on their own, inventing students were better prepared to learn this procedure from later direct instruction.

\section{A FOCUS ON CURRICULAR COHERENCE}

Although the approach of inventing with contrasting cases may help students see important features of Faraday's law, the example given is subject to the criticism that it treats the concept as an isolated, empirical fact. It has been argued that this lack of connection between various topics is common to how students perceive a traditionally taught physics curriculum. As Brewe puts it, "In the standard approach the curriculum is organized such that new content is introduced in distinct chapters which atomizes the curriculum and leads students to miss the coherence of physics" [17].

Although experts may value coherence between ideas in physics, studies of traditional physics instruction have found that (1) students do not develop and apply wellstructured physics knowledge [18,19] and (2) students can come to view physics as a set of disconnected facts rather than a coherent set of ideas [20,21]. A common theme between many PER-based instructional efforts has been the explicitly stated goal of better fostering coherence of physics knowledge $[5,17,20,22,23]$. Such instructional efforts have been shown to be more successful than traditional ones at supporting conceptual learning outcomes [24,25].

In this set of studies, we select one particular approach to fostering conceptual coherence: using previously learned 
concepts to build new ones. This approach attempts to build coherence through explicit connections between physics concepts. The central question of this research is how building on prior concepts compares to instruction that uses contrasting cases to focus only on the concept to be learned.

\section{OUR STUDIES}

We compare contrasting cases instruction to instruction building new concepts from previously learned ones in a physics classroom setting, using the topic of Faraday's law. To be clear, in these studies, we use Faraday's law to refer to the "flux rule:" $\mathcal{E}=N\left|d \Phi_{B} / d t\right|$. Even though this is not identical to the Maxwell-Faraday equation, $\vec{\nabla} \times \vec{E}=-\frac{\partial \vec{B}}{\partial t}$, we focus on the flux rule for two reasons [26]. First, at the introductory level, Faraday's law is commonly taught as the flux rule. Second, this definition of Faraday's law aligns with what the students in our studies were learning and with the instructional materials adapted for this study.

As noted previously, it can be argued that both building on prior concepts and contrasting cases instruction have pedagogical benefits, albeit quite different ones. For the Faraday's law flux rule, one approach is to motivate induced currents through the Lorentz force. Some potential benefits of building on prior concepts here are (i) highlighting the coherence between different $\mathrm{E} \& \mathrm{M}$ concepts, (ii) providing students with multiple tools for understanding induced currents, and (iii) helping students see when Faraday's law is needed over the Lorentz force-for cases where the current is induced by a changing magnetic field (see Table I). On the other hand, contrasting cases of a phenomenon can help students look beyond the surface to see important features related to correctly applying the Faraday's law flux rule-for example, identifying whether or not there is a change in magnetic flux.

This research builds most directly on previous work by Shemwell, Chase, and Schwartz [11] on the benefits of learning Faraday's law through contrasting cases in a physics classroom setting. They found that looking across a set of cases to invent a general explanation was more effective than explaining each case in isolation for discovering Faraday's law. Specifically, students explaining each case separately were more likely to rely on case-specific features rather than a unified, general explanation. Our study again investigates the efficacy of explaining a set of contrasting cases for learning Faraday's law, but compares it to the common instructional practice of building on previously learned concepts. We also investigate the effect of these activities on longer term learning trajectories by studying the impact on subsequent learning from direct instruction on Faraday's law and subsequent topics, such as Lenz's law.

Additionally, we use these materials to investigate another issue: student enjoyment and self-assessments of learning with interactive engagement methods. Studies in PER have shown that instructional approaches that produce large learning gains are not necessarily enjoyed by students $[25,27,28]$. Student evaluations of teaching are a major component of how institutions and instructors evaluate teaching effectiveness [29], so low student enjoyment of these methods could potentially raise barriers to sustained implementation and evaluations of instructional success.

\section{STUDY 1}

Study 1 compared Faraday's law instruction using contrasting cases (CC) to instruction building on prior concepts (BPC) - in this case, the relevant prior concept is Lorentz force. We inserted both instructional approaches into small group discussion sections through an active learning style worksheet activity. The central questions were whether the instructional difference between the two activities would result in a difference in the students' ability to apply the Faraday's law flux rule, and whether any difference would be present on the final exam, covering both Faraday's law and subsequently learned, related topics.

\section{A. Research context}

This study was conducted in a large lecture $(N \sim 500)$, calculus-based, introductory electricity and magnetism course at a small, private university. The course was primarily taken by first and second year engineering students. Course meeting times consisted of three 50minute lectures and one 50-minute discussion section per week. There were 32 discussion sections, each run by a graduate teaching assistant (TA), with attendance in each capped at 18 students.

We deployed our instruction in discussion sections during week 7 of the 10-week academic quarter. At this time, the lecture had spent one class on Faraday's law, but

TABLE I. Principles for determining whether a current will be induced and the situations in which they make correct predictions in standard introductory physics courses.

\begin{tabular}{lcc}
\hline \hline & \multicolumn{2}{c}{ Correctly predicts if a current is induced when... } \\
\cline { 2 - 3 } Principle & Current induced by motion & Current induced by changing $B$ field \\
\hline Lorentz force: $\vec{F}=q \vec{v} \times \vec{B}$ & $\checkmark$ & $\checkmark$ \\
Faraday's law (flux rule): $\mathcal{E}=N\left|d \Phi_{B} / d t\right|$ & $\checkmark$ & $\checkmark$ \\
\hline \hline
\end{tabular}


students had not completed the homework or otherwise practiced using Faraday's law in class. 334 students completed the discussion section activity, completed a discussion section post-test, and had clicker data that provided information on preactivity lecture attendance $\left(n_{\mathrm{CC}}=177, n_{\mathrm{BPC}}=157\right)$. There were $16 \mathrm{TAs}$, who taught two discussion sections each. The TAs were split into two groups, 8 teaching with the $\mathrm{CC}$ materials and 8 teaching with the BPC materials. The research team ran the TA training meeting for that week, where the TAs were introduced to the materials and instructions for running the discussion section. The TAs were separated according to their two instructional groups to discuss their particular materials. Generally, TAs in both instructional groups were instructed to refrain from telling the students the answers and to encourage students to work together to answer the questions on the worksheet.

\section{B. Instructional materials: Building on prior concepts and contrasting cases}

Both sets of materials had two instructional goals related to Faraday's law: to prepare students (i) to predict whether or not a current would be induced by attending to the change in magnetic flux through the loop and (ii) to rank the magnitudes of the induced current by attending to how quickly the flux is changing. We briefly discuss the key features of these materials below. The complete instructional materials are provided as Supplemental Material [30].

\section{1. "Building on prior concepts" materials}

The BPC activity was adapted from the Tutorial in Introductory Physics on Lenz's law [8]. This activity prompted students to apply their knowledge of the Lorentz force to make predictions of whether or not there would be a current produced from the magnetic forces exerted on the charges in the wire, as shown in Fig. 2. The configurations included a stationary loop and a moving loop in a uniform external $B$ field, a stationary loop and a moving loop in the nonuniform $B$ field of a bar magnet, and two loops rotating in a uniform $B$ field at different rates. These cases were chosen to span a range of loop movements and external $B$ field configurations. Students answered these questions in sequence with no prompt directing them to make explicit comparisons between the different configurations. For all cases, there were no changing magnetic fields, currents were induced by motion of a wire loop, and a correct determination of whether there was an induced current could be made from the Lorentz force.

\section{Contrasting cases materials}

The CC instruction presented students with six cases simultaneously (Fig. 3) with the following instructions: "Considering all the cases above, write a general explanation for when there will be an induced current that explains all 6 cases." The cases were a subset of the cases given in the Lorentz force materials. Students were told whether or not there would be an induced current in each of the 6 cases. Although students had already seen the Faraday's law equation in lecture, the selection of the cases aimed to illustrate a range of variation that could help students look past the surface features to recognize the key feature: change in magnetic flux.

\section{Tell and practice: Qualitative Faraday's law}

After completion of either the BPC or CC activity, students were led through an explanation of Faraday's law by way of comparison to Lorentz force, which we describe below. Again, this explanation was adapted from the Tutorial in Introductory Physics on Lenz's law. Students were asked to predict whether there would be an induced current in a stationary loop within an increasing, uniform magnetic field (a) if they applied their prior reasoning (either their "invented" rule in CC or Lorentz force in BPC) and (b) if a change in magnetic flux leads to

(Work Together) Now suppose the loop is placed in the magnetic field of a bar magnet as shown. Determine whether there would be a current through the wire loop in each case below. Explain in terms of magnetic forces exerted on the charges in the wire of the loop [i.e. $\vec{F}=q(\vec{v} \times \vec{B})$ ].

- The loop is stationary

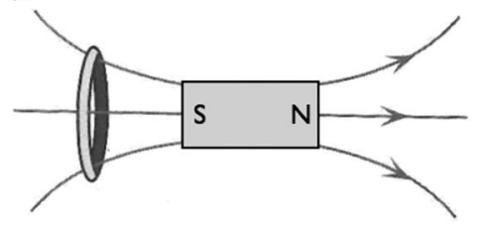

- The loop is moving to the right, towards the magnet.

- The loop is moving to the left, away from the magnet.

FIG. 2. An excerpt from the BPC materials. Students are asked to use the Lorentz force to predict whether there is an induced current. 

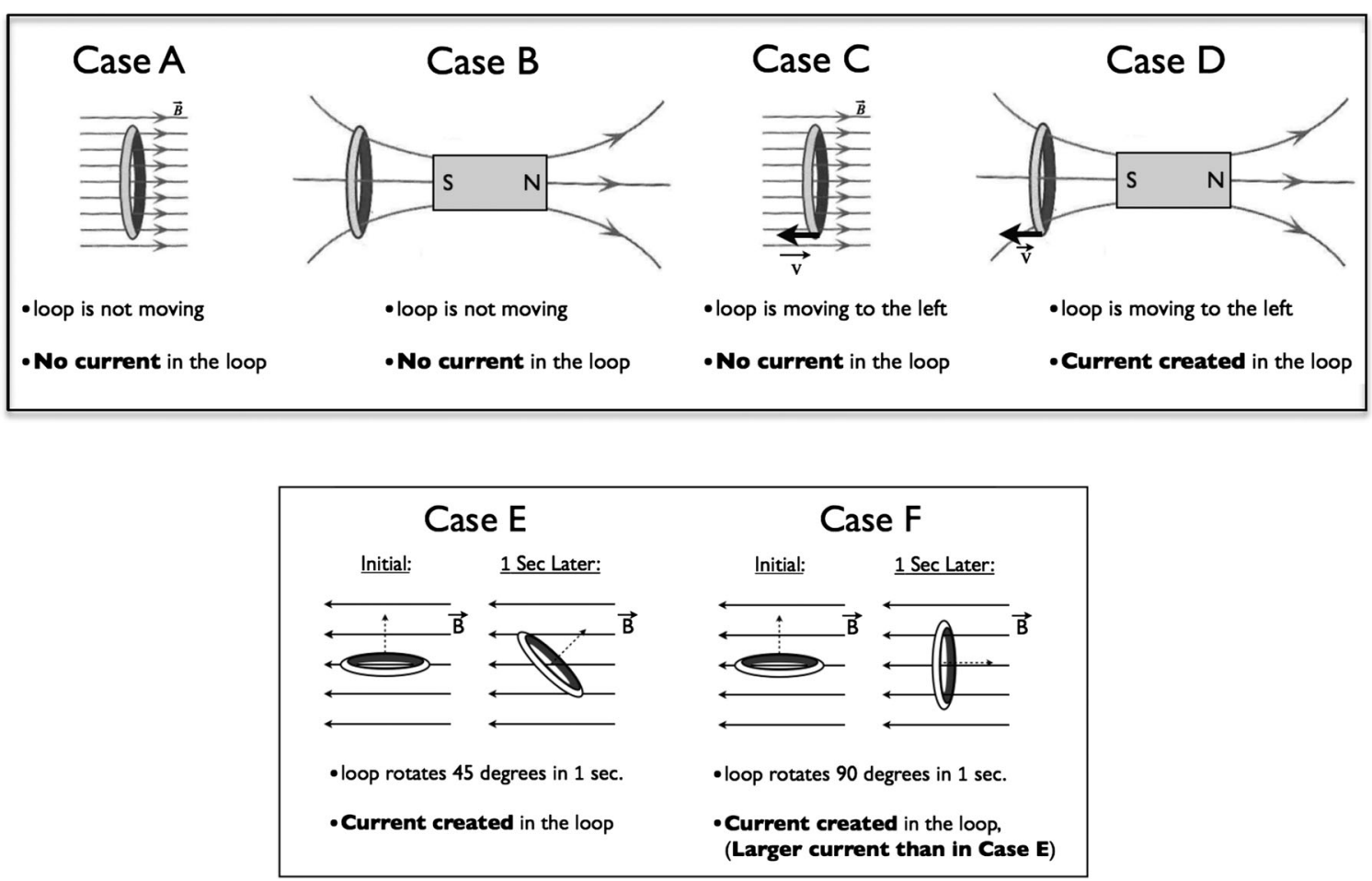

FIG. 3. The examples provided in the $\mathrm{CC}$ materials.

an induced current (if different from the rule invented in the CC activity).

The worksheet then stated that there will be an induced current in this case and provided a brief description of Faraday's law, highlighting two points. First, Faraday's law is a more broadly correct predictor of induced currents than Lorentz force, which can make correct predictions when current is induced by motion but not when current is induced by a changing $B$ field. Second, the qualitative description of Faraday's law consists of two statements:

- A change in the magnetic flux through the loop induces a current in the loop.

- The current is larger when the magnetic flux changes faster.

Following this, students were presented with the same cases from the previous activity, either CC or BPC. CC students were asked to check the given outcome with Faraday's law, and BPC students were asked to predict the outcome using Faraday's law instead of Lorentz force reasoning. Both groups were then asked to apply Faraday's law to rank the magnitudes of the induced currents in four new situations where the current is induced by a changing magnetic field.

The explicit description of Faraday's law here served two purposes. First, it provided a stand-alone, qualitative introduction to Faraday's law. Second, it provided an opportunity to enhance the learning from both activities. For contrasting cases, looking across the cases can help students differentiate important features of the phenomenon, preparing them to see how the statement of Faraday's law fits with these key features. For building on prior concepts, the activity is meant to highlight the coherence with Lorentz force and the need for the Faraday's law flux rule for cases where current is induced by a changing $B$ field. The main question is whether these two activities would differently prepare students to learn from direct instruction on Faraday's law.

\section{Research design}

Figure 4 shows the experimental timeline. First, one lecture introduced the Faraday's law flux rule as a rule that can correctly predict currents induced by motion or a changing magnetic field. In this lecture, the mathematical equation of Faraday's law was stated, $\mathcal{E}=N\left|d \Phi_{B} / d t\right|$, and the individual terms were explained. An explanation of electromotive force and a reminder of how to calculate flux followed. Then, the instructor solved a motional EMF problem (the classic problem of a metal bar sliding along two conducting rails in a static, uniform $B$ field) using both Faraday's law and the Lorentz force.

After this lecture, students attended the discussion section, where they worked in groups of 3-4 to either (i) use a set of contrasting cases to write an explanation for when a current is induced (CC) or (ii) use the Lorentz force to predict when there will be an induced current (BPC). Then, both groups were given the tell-and-practice materials for the qualitative features of Faraday's law. Finally, students individually answered a series of post-test questions to see if the two different instructional materials led to 


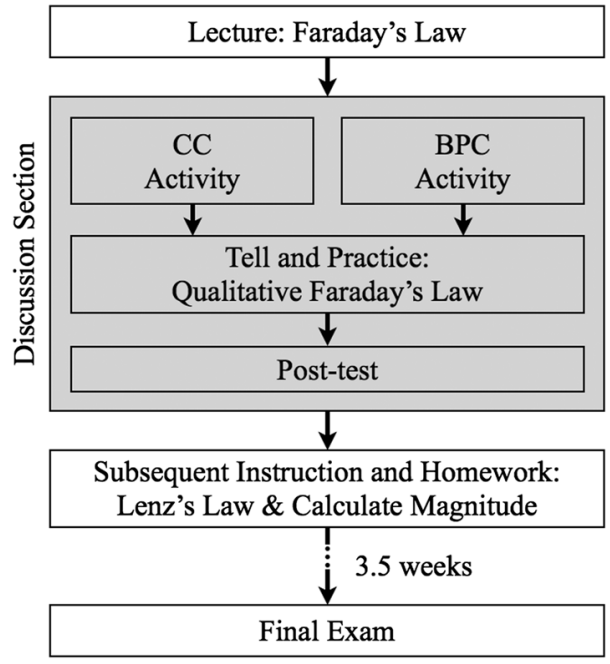

FIG. 4. Experimental timeline for study 1.

any differences in students' abilities to answer questions related to induced currents.

The post-test presented students with three problems that required them to predict whether a current would be induced in one situation, the relative magnitudes of induced currents, or the direction of the current. Finally, there were two 5-point Likert scale questions, asking students how much they enjoyed the discussion section activity and how much they learned from the discussion section activity. The complete post-test is given in the Supplemental Materials.

After the discussion sections, both sets of materials were made available to all students through the course website. This was due to the instructors' wishes for any long-term differences in instruction to be minimized. The next lecture covered Lenz's law. Students then completed a homework assignment that gave them practice in using Lenz's law to determine direction and Faraday's law to determine magnitude of induced currents for cases where current is induced by motion and cases where current is induced by a changing $B$ field. Then, one Faraday's law question on the final exam, given 3.5 weeks later, measured whether the instructional difference in the discussion sections had any long-term effect.

\section{Study 1 results}

\section{Post-test questions}

Summing students' scores on the induced current and magnitude questions, we obtained a Faraday's law post-test score (ranging from 0 to 3). There was no difference in post-test score by $\mathrm{CC}$ and $\mathrm{BPC}$ instructional materials $\left(m_{\mathrm{CC}}=1.82, s d_{\mathrm{CC}}=0.72, m_{\mathrm{BPC}}=1.88, s d_{\mathrm{BPC}}=0.70\right)$, $F(1,330)<0.1$.

However, a subsequent content analysis of the preceding lecture revealed that, unbeknownst to the research team, the lecture directly covered two of the post-test questions.

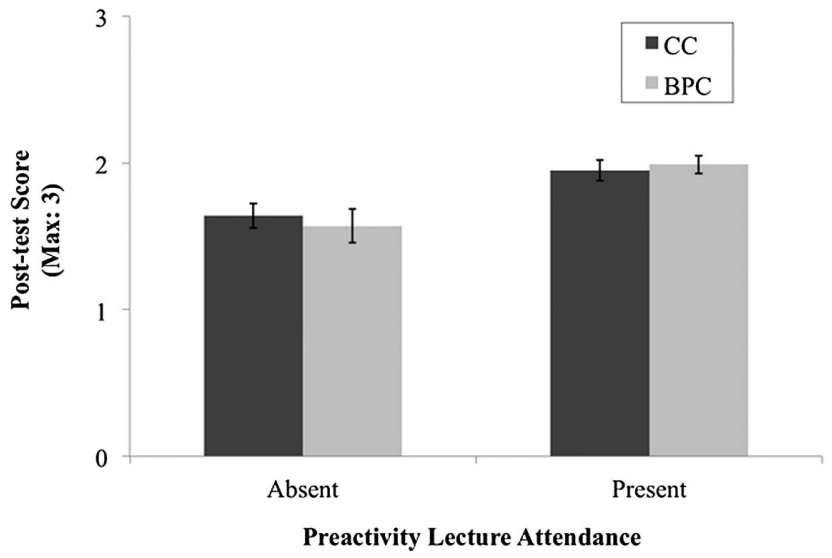

FIG. 5. Mean post-test score, split by instructional materials and preactivity lecture attendance. There is no performance difference by instructional materials, but there is an advantage for attending lecture. All error bars denote one standard error.

Students who attended the preactivity lecture had a higher post-test score than those who did not, $F(1,330)=20.2$, $p<0.001$, as shown in Fig. 5. We hypothesize that because the lecture directly covered two questions on the post-test, for students who attended lecture the posttest was a measure of their memory of a specific case. Thus, the post-test was not a good measure of students' understanding of Faraday's law more broadly.

There was no interaction of activity by preactivity lecture attendance, $F(1,330)=0.40, p=0.53$, so attending lecture had the same effect on students' post-test scores, independent of which instructional materials they later received in the discussion section.

\section{Enjoyment and self-assessment of learning}

On the enjoyment question, there was a significant effect of condition, where students exposed to contrasting cases ranked their enjoyment higher $\left(m_{\mathrm{CC}}=3.33\right.$, $\left.s d_{\mathrm{CC}}=0.95, m_{\mathrm{BPC}}=3.00, s d_{\mathrm{BPC}}=1.03\right), \quad F(1,327)=$ $5.60, p=0.019$, but there was no effect of lecture attendance, $F(1,327)=0.16, p=0.69$, and no interaction between the two factors, $F(1,327)=1.63, p=0.20$. So even though there was no difference in post-test scores by condition, students enjoyed the $\mathrm{CC}$ materials more.

On the learning question, there was a marginal effect of condition in favor of contrasting cases $\left(m_{\mathrm{CC}}=3.53\right.$, $\left.s d_{\mathrm{CC}}=0.95, m_{\mathrm{BPC}}=3.32, s d_{\mathrm{BPC}}=1.05\right), \quad F(1,327)=$ $3.29, p=0.07$. Again, there was no effect of lecture attendance, $F(1,327)=0.24, p=0.63$, and no interaction between the two factors, $F(1,327)<0.1$. Notably, students' self-assessment of their learning did not correlate with their post-test score, $r=0.05, p=0.40$, but did correlate with their enjoyment, $r=0.66, p<0.001$. This is consistent with prior research showing that selfassessments of competence do not match actual measures of performance [31] and that these self-assessments of 
learning are more highly correlated with affective measures than performance measures [32].

\section{Final exam question}

The final exam question assessed whether the instructional difference in the discussion sections would make a difference 3.5 weeks later, on content beyond what was covered in the discussion section activity-specifically, using Lenz's law to determine the direction of the induced current and magnetic field. The relevant final exam question [33] is shown here:

A metal ring of area $A=10 \mathrm{~cm}^{2}$ and electric resistance $R_{0}=2 \Omega$, mounted on a wood stand, is immersed in a uniform magnetic field $B$ that initially points into the page. The field's strength varies as shown in the graph (Fig. 6). Positive B means the magnetic field points into the page while negative $B$ means it points out of the page.

(a) (3 points) At $t=1 \mathrm{~s}$, is the current around the ring clockwise, counterclockwise, or zero? Explain.

(b) (3 points) At $t=3 \mathrm{~s}$, what is the direction of the magnetic field created by the current in the ring at the center of the ring? Is it into the page, out of the page, or zero? Explain.

(c) (3 points) At $t=5 \mathrm{~s}$, is current flowing around the ring in the same direction it was flowing at $t=3 \mathrm{~s}$ ? Explain.

(d) (6 points) Calculate the magnitude of the current flowing around the ring at $t=3 \mathrm{~s}$.

Twelve out of the 15 points of this question was for material not covered by the CC and BPC materials-parts (b) and (c) require Lenz's law and part (d) requires numerical calculation. Additionally, following our discussion section intervention, students in both groups had a lecture covering Lenz's law, a subsequent homework assignment giving students practice with applying Faraday's and Lenz's laws, and opportunity to study this material for the final exam. Given that the CC and BPC activities did not cover these later concepts and that both groups received identical instruction afterwards, it could be predicted that $\mathrm{CC}$ and $\mathrm{BPC}$ students would perform equally well on this final exam question.
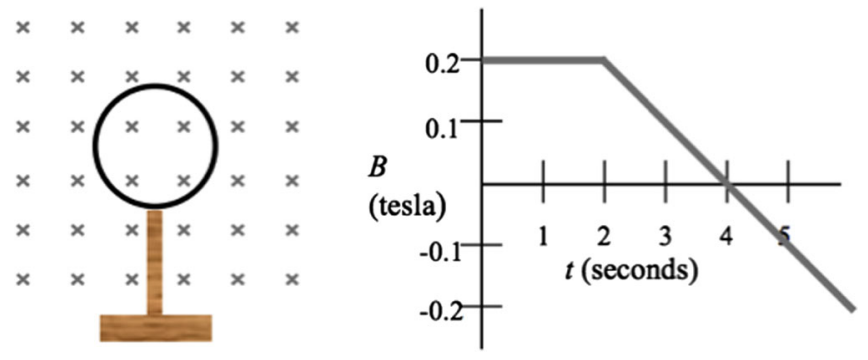

FIG. 6. Diagram of a metal ring in an external magnetic field, along with a graph of external magnetic field vs time.

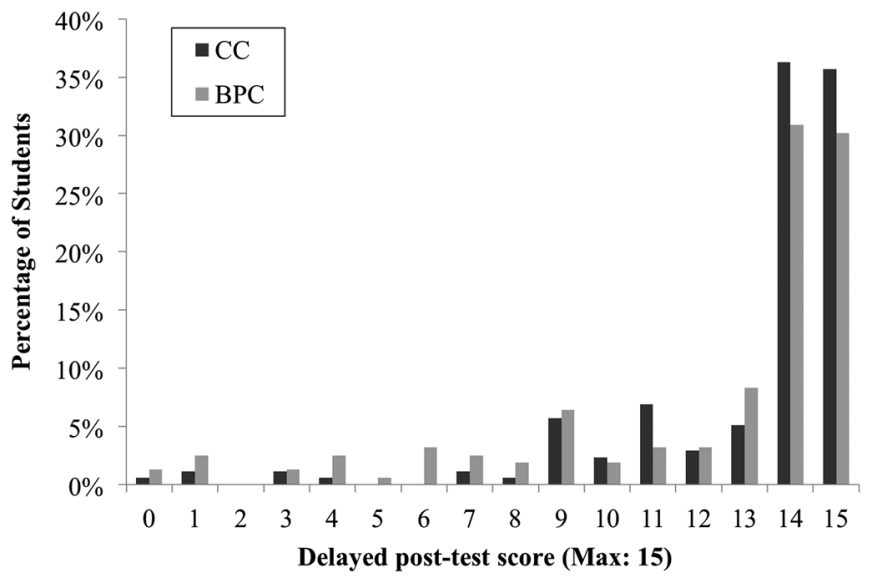

FIG. 7. The distribution of scores on the delayed post test for $\mathrm{CC}$ and $\mathrm{BPC}$. The difference between the distributions is reflected in the low tail for BPC and the greater ceiling effect for $\mathrm{CC}$ at scores of 14 and 15.

However, another plausible prediction, favoring the benefits of contrasting cases, is that by having students examine a set of contrasting cases of loops in magnetic fields, students could differentiate important, subtle features-such as the difference between amount of flux and change in flux. This could prepare students to learn from subsequent instruction, even for different, though related, topics. For example, an easy mistake to make in applying Lenz's law is to state that the induced magnetic field opposes the flux rather than the change in flux. Differentiating amount of flux and change in flux may help students more accurately apply Lenz's law. Note that parts (b) and (c) of this question target this plausible mistake.

The scores on the delayed post-test show a difference in the distributions for CC and BPC, shown in Fig. 7, with CC students scoring higher than BPC students, Mann-Whitney $U(332)=11987, Z=2.08, p=0.037$. Importantly, CC and BPC students' scores did not differ on the other final exam short answer questions, Mann-Whitney $U(332)=$ $13579, Z=0.18, p=0.86$, indicating that the difference was due to the intervention rather than generally higher performance in the course for the CC group.

As shown in Fig. 7, the difference in distributions is most noticeable in the low tail for the BPC students and the greater ceiling effect for $\mathrm{CC}$ at scores of 14 and 15 . Because of this strong ceiling effect, it is impossible to say whether the benefit of $\mathrm{CC}$ is primarily for the low performing students or if we simply are unable to measure the benefit for the high performing students. If the latter is true, that actual average benefit would be greater than reported here.

\section{E. Discussion}

The advantage of $\mathrm{CC}$ on the delayed post-test is evidence for the benefits of contrasting cases. This advantage was especially notable given the small instructional difference among repeated opportunities to learn and practice using 
Faraday's law and Lenz's law. This result is consistent with our prediction that contrasting cases helped students attend to and understand key features of the phenomenon, such as change in magnetic flux. The question remains as to whether this focus on recognizing key features of induced currents helped students simply learn how to predict whether there will be an induced current, or whether it helped them in learning subsequent, related ideas. For example, attention to the change in flux could help students use Lenz's law, because the direction of the induced magnetic field opposes that change in flux. Because we did not have access to students' scores for the separate subparts of the final exam question, we could not answer this question.

Additionally, the majority of the final exam question covered content learned between our intervention and the final exam, so one uncontrolled factor that impacted this outcome is the instruction and practice students experienced with induced current concepts in the 25 days between the discussion section activity and the final exam.

Because the post-test questions covered situations directly presented in lecture, we argue that the post-test was, for students who attended lecture, a test of memory rather than of comprehension of Faraday's law. Because of this, we cannot make any conclusions about student understanding of Faraday's law from the post test alone.

There are multiple possible explanations for the difference in enjoyment of the CC and BPC activities. One possibility is that these students enjoy the less structured explanation task in the $\mathrm{CC}$ materials more. In contrast, the BPC materials constrain students to answer specific questions with a specific concept. Another possibility comes from TA reports that the $\mathrm{CC}$ materials took less time to complete than the BPC materials. This difference could be explained by the additional examples in the BPC materials (8 situations instead of 6 in CC) and by the fact that BPC students had to determine whether there would be an induced current for each situation, as opposed to CC students who were told whether each loop had an induced current. A third possibility is that BPC students expecting to receive instruction and practice on Faraday's law were dissatisfied by an activity ostensibly covering Lorentz force.

\section{STUDY 2}

To address this question of whether the contrasting cases simply helped students better apply Faraday's law or whether attention to important features, such as change in flux, improved students' ability to understand subsequent topics as well, study 2 augmented our previous intervention with instruction on Lenz's law and calculating the magnitude of the induced current. This allowed us to standardize student instruction on these subsequent topics. We also expanded the post-test to include both topics covered differently by the CC and BPC activities (i.e., a qualitative understanding of Faraday's law) as well as subsequent content covered in the same way for both groups (i.e., Lenz's law), being careful to avoid questions directly covered in lecture.

Additionally, to discount shorter length as a possible explanation for the greater enjoyment of the contrasting cases materials, the number of situations seen in the two materials was made equal.

\section{A. Research context}

Study 2 took place during another iteration of the same physics course as in study 1 , which was taught by the same instructor the following year. In this iteration, the length of the discussion sections was increased from 50 to 75 minutes. Another difference from study 1 was that two lectures on Faraday's law preceded the discussion sections instead of just one. However, due to the slower pace of this iteration of the course, the two lectures covered comparable material to the one lecture in study 1 .

Additionally, to address potential differences in TA instruction, we had each TA teach one section with the $\mathrm{CC}$ materials and one section with the BPC materials, counterbalancing the order randomly across the TAs. Enrollment in this course was lower, so there were only 30 discussion sections with 15 TAs. 309 students completed the activity and post-test in their discussion section $\left(n_{\mathrm{CC}}=151, n_{\mathrm{BPC}}=158\right)$.

\section{B. Timeline and materials}

The new experimental timeline is shown in Fig. 8. The increased length of the discussion sections allowed us to cover additional topics. The complete activities are provided as Supplemental Material. The main changes to the materials are described here.

\section{Changes to study 1 materials}

As compared to CC, the BPC materials in study 1 included two additional examples of wire loops in motion. In study 2 , we removed these additional examples so that the CC and BPC materials included exactly the same cases, making the lengths of the activities more similar. This

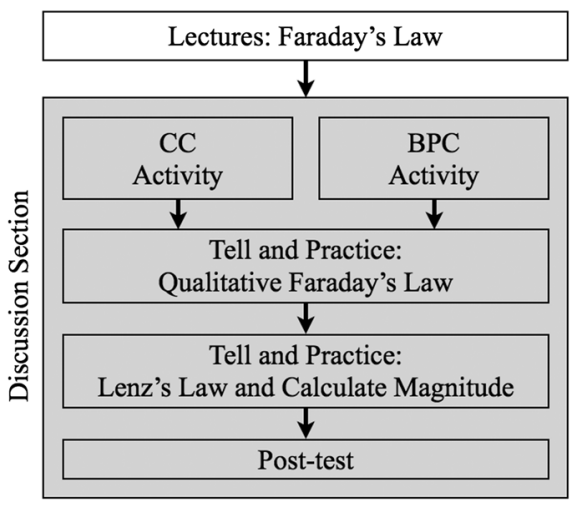

FIG. 8. Experimental timeline for study 2. 
change was also reflected in the tell-and-practice materials for Faraday's law, which asks students to apply Faraday's law to the same cases.

\section{Tell and practice: Lenz's law and calculate magnitude}

In response to study 1, we added tell-and-practice instruction on Lenz's law and calculating the magnitude of the induced current. This allowed us to standardize the instruction on these subsequent topics, in order to assess the impact of $\mathrm{CC}$ and BPC activities on this subsequent learning.

CC and BPC students received identical instruction, which included an explanation of the relevant principle (either Lenz's law or the quantitative statement of Faraday's law), a worked example, and four practice problems (provided in the Supplemental Materials). All of these problems presented cases where the Faraday's law flux rule must be used, because the current is induced by a changing magnetic field. Students worked together in groups to answer these problems.

\section{Changes to the post-test}

The post-test was expanded, probing student performance on qualitative Faraday's law questions as well as mastery of subsequent topics, such as Lenz's law and calculating the magnitude of the induced current. The post test broke down as follows:

Qualitative Faraday's law:

- "Zero current" (4 questions)—situations where there is a magnetic flux, but it is not changing, so there is no current.

- "Rank magnitudes" (1 question)—ranking the relative magnitudes of the induced currents in 4 different loops moving in a uniform magnetic field.

Subsequent Topics:

- "Lenz's law" (4 questions)—determining the direction of the induced current or induced magnetic field when it is nonzero.

- "Calculate magnitude" (1 question)—calculating the numerical answer for the magnitude of the induced current.

These changes allowed us to replicate the kinds of questions probed by the final exam problem in study 1 . Also, the subsequent instruction on Lenz's law and calculating the magnitude was standardized for the two groups. Both the qualitative Faraday's law and subsequent topics questions presented a mix of situations where there was either motion of the wire loop or a changing magnetic field.

\section{Post-test results}

We present the post-test results according to the two different groups of questions: (i) the "qualitative Faraday's law" questions and (ii) the "subsequent topics" for which both groups received identical instruction.

\section{Qualitative Faraday's law post-test}

First, we investigated differences in performance related to our $\mathrm{CC}$ and BPC instructional difference by looking at the number of correct answers to the qualitative Faraday's law post-test questions (ranging from 0 to 5 ). We performed a two-way ANCOVA with instructional materials and preactivity lecture attendance as the two independent variables, controlling for previous midterm score. Because there were two lectures before the discussion section activity, we split students according to whether they were absent from both lectures, or whether they attended at least one lecture. We chose this distinction, because we wanted to separate students who received some introduction to Faraday's law from students who did not. Twentyone students in the BPC instruction and 20 students from the $\mathrm{CC}$ instruction attended neither one of the lectures. Faraday's law post-test scores, broken out by instructional materials and lecture attendance, are shown in Fig. 9.

There was a main effect of instructional materials, $F(1,304)=8.99, p=0.003$, with better performance in CC. There was no main effect of lecture attendance, $F(1,304)=0.34, p=0.56$, but there was a marginal interaction between condition and lecture attendance, $F(1,304)=3.66, p=0.057$, indicating a larger performance gap between CC and BPC for students who did not attend lecture than for students who did attend.

Overall, there is better performance in the CC condition on the qualitative Faraday's law post-test. However, the interaction of instructional materials and lecture attendance is also important. Not surprisingly, students in the BPC condition that attended lecture do better (though not statistically significantly) than the BPC students that do not. More notable is that the CC students who did not

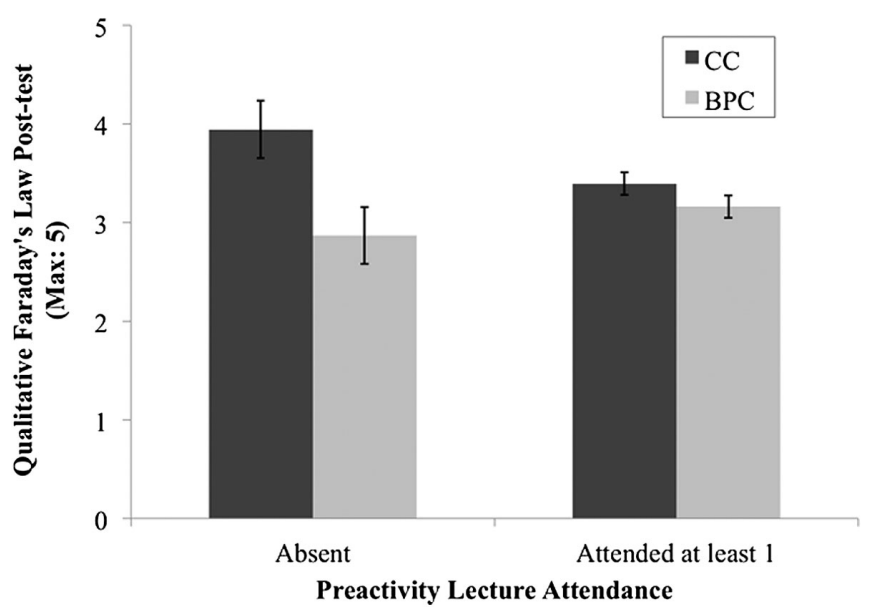

FIG. 9. Qualitative Faraday's law post-test scores, controlling for previous midterm score, broken out by instructional materials and lecture attendance. All error bars denote one standard error. 
attend lecture perform marginally better than $\mathrm{CC}$ students who do attend lecture $p=0.08,95 \% \mathrm{CI}$ of the difference $[-0.07,1.17]$. Although it seems unintuitive that students who had less instruction perform better, not having been told the Faraday's law flux rule may have increased search across the contrasting cases for a single explanation that determines induced current. This increased search could provide students with more opportunities to identify the different key factors involved in generating induced currents, increasing the benefits of the contrasting cases. Students who were told Faraday's law and verify that it makes the correct prediction for each case may not have experienced the full benefit of the contrasts between the cases. This is consistent with Schwartz et al., who found that inventing an explanation for a set of contrasting cases prior to direct instruction increased search across the different cases, compared to working with the cases when told the explanation beforehand [12].

\section{Subsequent topics post-test}

For the subsequent topics post-test scores (ranging from 0 to 5), we performed the same two-way ANCOVA as before. The subsequent topics post-test scores, broken out by instructional materials and lecture attendance and controlling for previous midterm score, are shown in Fig. 10. There was a main effect of instructional materials, $F(1,304)=4.12, p=0.043$, so even though the Lenz's law instruction was identical for both groups, the CC students were better prepared to learn from that instruction.

However, there was no difference by prediscussion lecture attendance, $F(1,304)=0.22, p=0.64$, and no interaction, $F(1,304)=1.09, p=0.30$. It makes sense that there would be no effect of lecture attendance, since these subsequent topics were not covered in the lecture.

We propose that the benefit of the contrasting cases for learning to attend to change in flux helps students learn and apply the subsequent topic of Lenz's law. One alternative

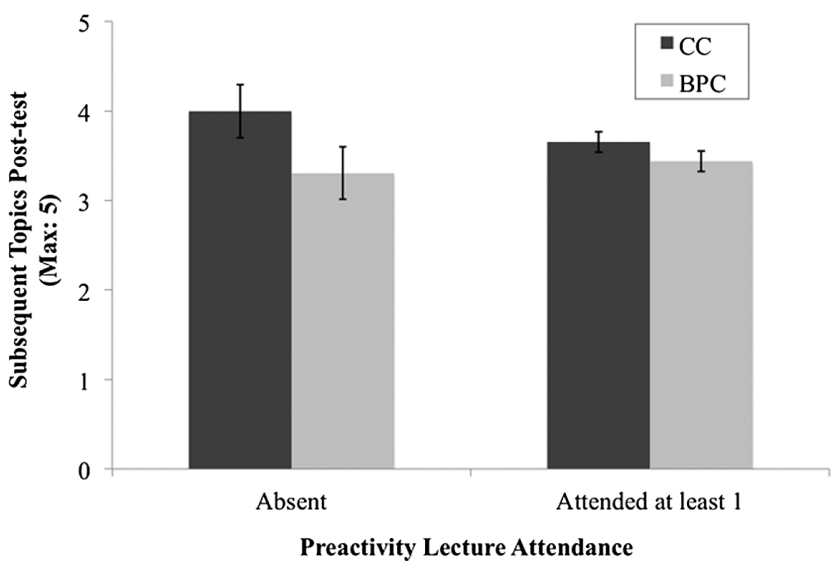

FIG. 10. Subsequent topics post-test scores, controlling for previous midterm score, broken out by instructional materials and lecture attendance. All error bars denote one standard error.
TABLE II. Percentage of responses stating that there is no induced current on the Lenz's law questions, split by instructional materials. There is no significant difference in percentage of "no current" responses by instructional materials.

\begin{tabular}{lcc}
\hline \hline Lenz's law & \multicolumn{2}{c}{ "No current" responses } \\
\cline { 2 - 3 } question & $\begin{array}{c}\text { Contrasting } \\
\text { cases }\end{array}$ & $\begin{array}{c}\text { Building on } \\
\text { prior concept }\end{array}$ \\
\hline 1 & $1 \%$ & $1 \%$ \\
2 & $1 \%$ & $0 \%$ \\
3 & $1 \%$ & $2 \%$ \\
4 & $11 \%$ & $13 \%$ \\
\hline \hline
\end{tabular}

explanation we can rule out is that the difference on the subsequent topics post-test simply reiterates the difference in correct application of Faraday's law. Specifically, BPC students could perform worse in identifying the direction of the current, because they incorrectly state that there is no induced current at all. However, looking at students' responses to the Lenz's law questions, we see that there is no difference in how many students incorrectly answer that there is "no current" (Table II). Therefore, the effect of the CC materials is actually an increase in correct predictions of the induced current direction.

\section{Enjoyment and self-assessment of learning}

In study 1 , one complicating factor was that the enjoyment question was asked after the post-test. Here in study 2, both the enjoyment and the self-assessment of learning questions were asked after the activity but before the posttest. Therefore, the results from study 2 are more likely to represent students' beliefs about the activities, independent of their experience with the post-test.

On the enjoyment question, a two-way ANOVA showed a significant effect of instructional materials, where students exposed to contrasting cases again ranked their enjoyment higher, $F(1,305)=12.8, p<0.001$, with a marginal effect of lecture attendance, $F(1,305)=3.23$, $p=0.07$, and a significant interaction between the two factors, $F(1,305)=6.26, p=0.013$. Mean enjoyment scores are shown in Fig. 11.

The interaction is driven by the especially low rankings of BPC students who did not attend lecture as compared to the rankings of the other three groups, $p<0.001,95 \% \mathrm{CI}$ interval for difference $[-1.24,-0.39]$. One explanation is that BPC students who do not attend the lecture cannot make sense of why the Faraday's law instructional activity starts with a review of Lorentz force. On the other hand, BPC students who did attend lecture learn about the link between Lorentz force and Faraday's law beforehand. For CC students, the discussion section deals directly with Faraday's law, which may match their expectations for a Faraday's law activity. 


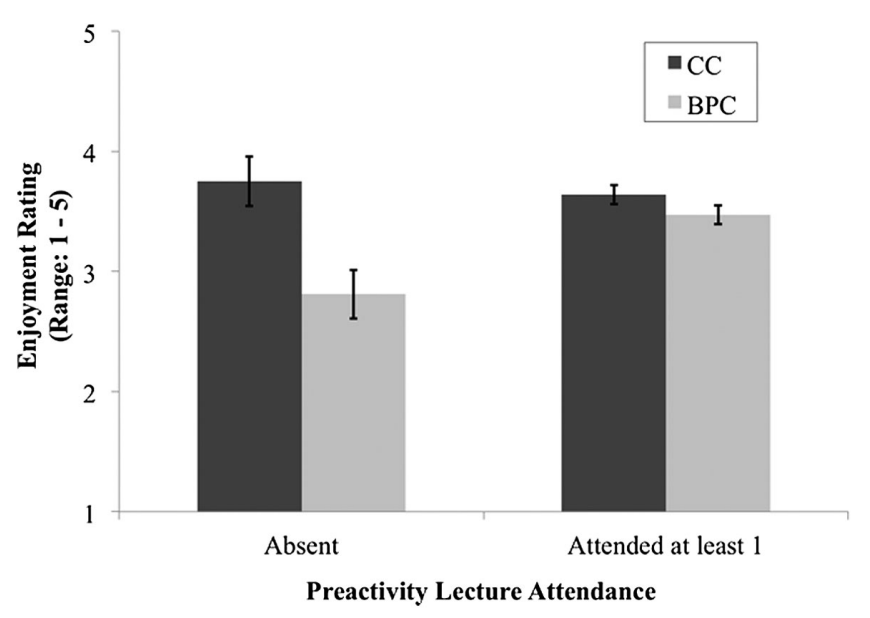

FIG. 11. Mean enjoyment ratings of the discussion section activities, split by materials and preactivity lecture attendance. All error bars denote one standard error.

On the learning question, there was a main effect of condition in favor of contrasting cases $\left(m_{\mathrm{CC}}=3.53\right.$, $\left.s d_{\mathrm{CC}}=0.95, m_{\mathrm{BPC}}=3.32, s d_{\mathrm{BPC}}=1.05\right), \quad F(1,305)=$ $4.21, p=0.013$. As in study 1 , there was no effect of lecture attendance, $F(1,305)<0.1$, and no interaction between the two factors, $F(1,305)=0.42, p=0.52$. Again, students' self-assessment of their learning did not correlate with their total post-test score, $r=0.03$, $p=0.65$, but did correlate with enjoyment, $r=0.47$, $p<0.001$.

In summary, the results show that $\mathrm{CC}$ students once again rated their enjoyment higher. Although the explanation is not clear, we can see that the result holds even when the lengths of the materials are made more equal. As before, self-assessment of learning was not correlated with posttest score, but it was correlated with enjoyment.

\section{Discussion}

Students who explored the contrasting cases perform better on both qualitative Faraday's law questions and subsequent topics questions. This supports our explanation that the benefits of the cases for understanding the key features, such as change in flux, can benefit learning of subsequent topics. In the contrasting cases activity, the search for a common explanation of induced currents may help students look past surface features that are insufficient to explain all the cases on their own, such as a nonzero magnetic flux or movement of the loop. This may help students recognize change in flux as the key feature, either when searching across the cases or in the subsequent description of Faraday's law.

Here, being absent from lecture may actually provide a benefit. Not knowing the correct answer may increase student search across the contrasting cases for a common explanation, increasing students' knowledge of the feature space, benefiting future learning of the concept.

\section{CONCLUSION}

\section{A. Benefits of inventing a general explanation with contrasting cases}

In summary, we have shown that a contrasting cases activity focused on developing a single explanation for multiple examples helps students learn to correctly apply a physics principle. Students also found this activity more enjoyable.

In other areas in physics, students have been found to have difficulty seeing change in a quantity rather than amount of a quantity $[34,35]$. Given these difficulties, the core benefit of the contrasting cases here could be in providing students with a need to slow down and notice the change in the amount of magnetic flux rather than just the amount of magnetic flux at a single point in time. The benefit for Lenz's law is then obvious, as determining the direction of the induced magnetic field relies on students' ability to determine how the flux is changing.

\section{B. Lack of observed value to developing coherence between concepts}

We have shown that practice explaining a set of contrasting cases of a new concept leads to better future application of related concepts than connecting the new concept to a previously learned one. While expertise in physics requires the development of coherence between the different concepts of physics, instruction emphasizing this connection did not help students apply these principles on typical problems over focused instruction on the new concept.

It is possible that other assessments would show different results. For example, it is possible that coherence between different concepts would give students a way to check their answer, such as determining induced currents with both the Lorentz force and Faraday's law. This coherence could also aid in concept selection, giving students a way to evaluate the comparative benefits of using either Lorentz force or Faraday's law for a given problem. It is also possible that students need better grounding in the individual concepts themselves before the benefits of coherence between different concepts become apparent.

To be clear, these studies are not an indictment of instruction emphasizing coherence between concepts. Rather, it is a call for instructors and researchers to develop assessments that can make explicit the benefits of that coherence.

\section{Is attending lecture harmful?}

Our results show that contrasting cases instructional materials can improve performance in a typical large-lecture, introductory physics course. However, we found that students who search for a general explanation of a set of contrasting cases without attending prior lectures perform the best after instruction. This raises important 
questions about the function and instructional benefits of lecture in an introductory physics course.

Previous research has shown that the benefits of contrasting cases are realized when students transition between the different cases, comparing features in the search of a common explanation $[11,12]$. For students who are told the answer ahead of time, they may simply apply the known solution to the cases one at a time. This is consistent with our study 2 results: CC students performed better if they were absent from lecture, perhaps because not knowing the correct answer increased search across the contrasting cases for the common explanation of induced currents.

Our suggestion is not that lectures should be eliminated, but rather that careful coupling between the direct instruction in lectures and contrasting cases activities can enhance student learning. Other studies have also shown that direct instruction can be enhanced when preceded by activities that help students develop prior knowledge that prepares them for future learning $[12,15,16,36]$. One implication is increased attention to instructional sequencing, where activities that emphasize discovery and exploration are scheduled before related lectures. Another possibility is to incorporate these sequences within the progression of a lecture. Peer instruction-style questions typically used as practice with concepts or assessment of student understanding in large lectures could serve another role. Before direct instruction, a question could be posed to the class asking them to seek an explanation for a set of contrasting cases. The elaboration of this potential approach along with assessment of its effectiveness is a potential future direction for further integrating contrasting cases into typical instructional contexts.

One unanswered question is whether the direct instruction in lectures is hurting student performance or whether there are inconsistencies between the contrasting cases and lecture instruction that cause confusion when taken together. Our interpretation is that learning Faraday's law in lecture lessens the benefits of seeking one explanation to a group of contrasting cases. Although prior work by Schwartz and colleagues $[15,16]$ would suggest it unlikely, here we cannot rule out the possibility that lecture alone may help students as much as contrasting cases alone, and that it is the combination of lecture and the contrasting cases that confuses students. Future study designs of this type could include a "lecture only" control group, to help decide between these two interpretations.

\section{Comparative studies of interactive engagement materials}

This research is fundamentally about understanding the structure of activities in interactive-engagement classroom settings. We found that a small instructional change to how a series of examples are examined-either as cases to be explained or as practice applying a previously learned concept-produced a noticeable and lasting difference.
This provides us with research-based instructional guidance for how the design of instructional materials can affect learning outcomes, guidance beyond the overall principle of "interactively engaging" students.

Critically, we argue that the instructional findings here are more fine-grained than those of typical studies supporting the benefits of interactive engagement for two methodological reasons. First, such studies typically compare instruction over an entire semester, measuring differences in end-of-class performance, with either course performance or a standardized survey. As a consequence, conclusions can only be drawn between the overall course curricula and not any one component within them. In this way, whole approaches to teaching are compared, with the similarity between these reformed courses commonly coarsely bundled as active engagement. Second, comparisons are commonly made between very different instructional approaches, usually between a PER-based instructional method and a traditional one $[6,25,37]$. While PER-based methods commonly outperform traditional instruction, it is unclear how consequential and effective the individual elements of these different PER-based approaches are.

By investigating the efficacy of individual activities in the same interactive engagement classroom, we can start to identify important components of instructional materials that foster learning and performance. In this study, we showed that an instructional approach taken from cognitive psychology-finding a general explanation for a set of contrasting cases, particularly when well sequenced with direct instruction-led to increased performance over a common active engagement approach. However, our broader aim is not just to show the instructional efficacy of contrasting cases, but also to advocate for these kinds of instructional comparisons and the articulation of assessments that highlight the benefits of different instructional approaches.

It is through this lens of fine-grained instructional comparisons that we address the issue of instructional significance. These studies were not an attempt to fully optimize the instruction of Faraday's law to obtain the greatest learning possible. Rather, it was a study of the impact a small change in instruction can make when embedded in a larger instructional context that includes lectures, homework, and exams. We somewhat arbitrarily chose the topic of Faraday's law as a test case for this research. What is notable is that this relatively small instructional difference led to any long-term effect, however small.

Take, for example, the qualitative Faraday's law post-test results of study 2, which showed the largest difference between performance for CC and BPC students. While the difference in scores for CC and BPC students who did not attend lecture was 1.1 points out of 5, for the majority of students who did attend lecture, the difference in scores was only 0.24 points out of 5 , or $5 \%$ of the total score. Obviously, instructors may not wish to make major 
adjustments to their teaching based on this small gain, but when instructors or physics education researchers are designing an instructional unit, such results should at least be considered. Our results indicate that a relatively small instructional difference, one that might be left up to an instructor's preference in many active learning approaches, makes a difference in subsequent student performance. We argue that this finding is educationally significant not because it immediately suggests a path to drastic gains in student learning, but because it suggests new avenues of research for improving on currently accepted best practices in teaching, not just for Faraday's law, but for many other topics in physics as well.

Our goal is to move toward research-based findings that can provide guidance on the design of instructional materials. By better illustrating the effects of the details of instructional materials, physics education researchers can start to clarify and explicate the design principles behind successful materials as well as provide guidance for the design of future materials, bringing science to the art of instructional design.

\section{ACKNOWLEDGMENTS}

We would like to thank the instructional staff of the physics course- the lecturer, the course coordinator, and the TAs-for working with us and allowing us to conduct this research. We would also like to thank members of the AAALab and Wieman Research Group for useful feedback on the research design and data analysis, especially Dan Schwartz for detailed guidance on the design of the instructional materials. Tyler Brown, Engin Bumbacher, Dilia Olivo, and Great Thavapatikom greatly assisted with logistics and data collection.
[1] I. A. Halloun and D. Hestenes, The initial knowledge state of college physics students, Am. J. Phys. 53, 1043 (1985).

[2] D. P. Maloney, T. L. O'Kuma, C. J. Hieggelke, and A. Van Heuvelen, Surveying students' conceptual knowledge of electricity and magnetism, Am. J. Phys. 69, S12 (2001).

[3] R. K. Thornton and D. R. Sokoloff, Assessing student learning of Newton's laws: The force and motion conceptual evaluation and the evaluation of active learning laboratory and lecture curricula, Am. J. Phys. 66, 338 (1998).

[4] P. R. L. Heron, Empirical investigations of learning and teaching. Part II: Developing research-based instructional materials, in Proceedings of the International School of Physics "Enrico Fermi," Course CLVI, edited by E. F. Redish and M. Vicentini (IOS Press, Amsterdam, 2004), pp. 351-366.

[5] L. C. McDermott, Oersted medal lecture 2001:'Physics Education Research - the key to student learning', Am. J. Phys. 69, 1127 (2001).

[6] R. R. Hake, Interactive-engagement versus traditional methods: A six-thousand-student survey of mechanics test data for introductory physics courses, Am. J. Phys. 66, 64 (1998).

[7] E. Kuo and C. E. Wieman, Seeking instructional specificity: an example from analogical instruction, Phys. Rev. ST Phys. Educ. Res. 11, 020133 (2015).

[8] L. C. McDermott, P. Shaffer, and the Physics Education Group at the University of Washington, Tutorials in Introductory Physics (Prentice Hall, Upper Saddle River, NJ, 2002).

[9] J. D. Bransford, J. J. Franks, N. J. Vye, and R. D. Sherwood, New approaches to instruction: Because wisdom can't be told, in Similarity and Analogical Reasoning, edited by S. Vosniadou and A. Ortony (Cambridge University Press, New York, 1989), pp. 470-497.
[10] S. Salehi, M. Keil, E. Kuo, and C. E. Wieman, How to structure an unstructured activity: Generating physics rules from simulation or contrasting cases, in 2015 PERC Proceedings, edited by A. D. Churukian, D. L. Jones, and L. Ding (College Park, MD, 2015), p. 291.

[11] J. T. Shemwell, C. C. Chase, and D. L. Schwartz, Seeking the general explanation: A test of inductive activities for learning and transfer, J. Res. Sci. Teach. 52, 58 (2015).

[12] D. L. Schwartz, C. C. Chase, M. A. Oppezzo, and D. B. Chin, Practicing versus inventing with contrasting cases: The effects of telling first on learning and transfer, J. Educ. Psychol. 103, 759 (2011).

[13] M. Chi, I. Dohmen, J. T. Shemwell, D. B. Chin, C. C. Chase, and D. L. Schwartz, Seeing the forest from the trees: A comparison of two instructional models using contrasting cases, in 2012 Annual Meeting of the American Educational Research Association (Vancouver, BC, Canada, 2012), pp. 1-10.

[14] J. D. Bransford and D. L. Schwartz, Rethinking transfer: A simple proposal with multiple implications, Rev. Res. Educ. 24, 61 (1999).

[15] D. L. Schwartz and T. Martin, Inventing to Prepare for Future Learning: The Hidden Efficiency of Encouraging Original Student Production in Statistics Instruction, Cognit. Instr. 22, 129 (2004).

[16] D. L. Schwartz and J.D. Bransford, A time for telling, Cognit. Instr. 16, 475 (1998).

[17] E. Brewe, Modeling theory applied: Modeling Instruction in introductory physics, Am. J. Phys. 76, 1155 (2008).

[18] M. T. H. Chi, P. J. Feltovich, and R. Glaser, Categorization and representation of physics problems by experts and novices*, Cogn. Sci. 5, 121 (1981).

[19] R. J. Dufresne, W. J. Gerace, P. T. Hardiman, and J. P. Mestre, Constraining novices to perform expertlike 
problem analyses: Effects on schema acquisition, J. Learn. Sci. 2, 307 (1992).

[20] E. F. Redish, J. M. Saul, and R. N. Steinberg, Student expectations in introductory physics, Am. J. Phys. 66, 212 (1998).

[21] W. K. Adams, K. K. Perkins, N. S. Podolefsky, M. Dubson, N. D. Finkelstein, and C. E. Wieman, New instrument for measuring student beliefs about physics and learning physics: The Colorado Learning Attitudes about Science Survey, Phys. Rev. ST Phys. Educ. Res. 2, 010101 (2006).

[22] A. Van Heuvelen, Learning to think like a physicist: A review of research-based instructional strategies, Am. J. Phys. 59, 891 (1991).

[23] R. Chabay and B. Sherwood, Restructuring the introductory electricity and magnetism course, Am. J. Phys. 74, 329 (2006).

[24] M. A. Kohlmyer, M. D. Caballero, R. Catrambone, R. W. Chabay, L. Ding, M. P. Haugan, M. J. Marr, B. A. Sherwood, and M. F. Schatz, Tale of two curricula: The performance of 2000 students in introductory electromagnetism, Phys. Rev. ST Phys. Educ. Res. 5, 020105 (2009).

[25] N. D. Finkelstein and S. J. Pollock, Replicating and understanding successful innovations: Implementing tutorials in introductory physics, Phys. Rev. ST Phys. Educ. Res. 1, 010101 (2005).

[26] For further discussion on the difference between Faraday's law and the flux rule, see R. P. Feynman, R. B. Leighton, and M. Sands, The Feynman Lectures on Physics, Vol. 2: Mainly Electromagnetism and Matter (Addison-Wesley, Reading, MA, 1979). Section 17-1 shows how the flux rule encompasses two distinct physical mechanisms, and section 17-2 provides some special cases where Faraday's law and the flux rule are not equivalent.
[27] P. W. Laws, Millikan Lecture 1996: Promoting active learning based on physics education research in introductory physics courses, Am. J. Phys. 65, 14 (1997).

[28] C. Turpen, N. D. Finkelstein, and S. J. Pollock, Towards understanding classroom culture: Students' perceptions of tutorials, AIP Conf. Proc. 1179, 285 (2009).

[29] C. Henderson, C. Turpen, M. Dancy, and T. Chapman, Assessment of teaching effectiveness: Lack of alignment between instructors, institutions, and research recommendations, Phys. Rev. ST Phys. Educ. Res. 10, 010106 (2014).

[30] See Supplemental Material at http://link.aps.org/ supplemental/10.1103/PhysRevPhysEducRes.12.010128

for the full instructional materials and assessment questions used in studies 1 and 2 .

[31] J. Kruger and D. Dunning, Unskilled and unaware of it: how difficulties in recognizing one's own incompetence lead to inflated self-assessments, J. Pers. Soc. Psychol. 77, 1121 (1999).

[32] T. Sitzmann, K. Ely, K. G. Brown, and K. N. Bauer, Selfassessment of knowledge: a cognitive learning or affective measure?, Acad. Manag. Learn. Educ. 9, 169 (2010).

[33] A. Elby (private communication).

[34] R. J. Beichner, Testing student interpretation of kinematics graphs, Am. J. Phys. 62, 750 (1994).

[35] A. F. Heckler and T. M. Scaife, Patterns of Response Times and Response Choices to Science Questions: The Influence of Relative Processing Time, Cogn. Sci. 39, 496 (2015).

[36] M. Kapur, Productive Failure in Learning Math, Cogn. Sci. 38, 1008 (2014).

[37] L. Deslauriers, E. Schelew, and C. Wieman, Improved learning in a large-enrollment physics class, Science 332, 862 (2011). 